\title{
Preparation and Characterization of Modified Silica-Epoxy Hybrid Ceramic Coatings
}

\author{
Mahmoud Y. Zorainy ${ }^{1, a}$ Ahmed Nashaat ${ }^{1}$, Yasser El-Shaer ${ }^{1}$, Mohamed Gobara ${ }^{1}$ and Ahmed Elbeih ${ }^{1}$ \\ ${ }^{1}$ Military Technical College, Kobry Elkobbah, Cairo, Egypt
}

\begin{abstract}
Hybrid sol-gel processing of inorganic-organic nanocomposites has been of a great interest over the last decades for being advantageous compared to the conventional addition methods of nanoparticles. In this study, a three-component system was adopted experiencing the design and preparation of different hybrid ceramic coatings based on Diglycidyl ether of Bisphenol A (Epoxy) and 3-Glycidyloxypropyl trimethyloxysilane (GLYMO) by sol-gel technique. The obtained hybrid coatings were cured using different hardeners, Diethylene triamine (DETA) as an organic linker and 3-Aminopropyl triethoxysilane (APTES) as an inorganic/organic linker. Microstructure assessment and the morphology of the prepared hybrids was investigated using FTIR and scanning electron microscopy (SEM) respectively. Mechanical properties (adhesion, and hardness) were determined. The degree of hydrophilicity of the hybrids was assigned depending on the contact angle measurements. Moreover, the thermal properties were investigated using thermogravimetric analysis (TGA). The results showed that the silica content plays an important role in determining the morphology as well as the mechanical, physical, and thermal properties of the coatings. The results showed an improvement in most of the properties of the hybrid coatings with increasing the silica content up to a certain extent. However, further increase in the silica content, leads to a clear deterioration.
\end{abstract}

\section{Introduction}

Sol-gel method is a low temperature processing technique that is used for the synthesis of the ceramic materials. A hybrid organic/inorganic has been thought of in order to achieve a homogeneous combination of both materials or somehow control their properties. In particular, silicon based hybrid materials using sol-gel processing is one of the major categories. The reason for the special role of silicon is the stability of the $\mathrm{Si}-\mathrm{C}$ bond of the coupling agents used during the formation of the silica that in turns, contributes in the formation of these hybrid networks [1]. In general, coatings have developed side by side with ceramics until a crossroad was once noticed. Ceramic coating has been of great interest, where a specific material with certain properties is being selected to adhere to a surface (substrate) showing the desired properties of ceramics or a synergistic character of both ceramics and the substrate. Hybrid coatings compromise two components; an organic one that is responsible for toughness, adhesion and elasticity. While the other is inorganic, that characterizes some other properties like hardness and wear resistance. Silica sol-gel coatings form van der Waals bonds with the metallic substrate that transforms into a stable covalent bond during the curing process. Epoxy-silica coatings were studied by a number of researchers. Generally, epoxy resins have superior chemical and corrosion resistance, high adhesion, and curability at ambient temperature. However, they suffer from poor thermal and weathering stabilities, reduced mechanical properties and unsuitable cutting properties. This limits the epoxy resins in highperformance applications [2]. Using silica inorganic precursor within the epoxy resins to form epoxy-silica hybrid materials may improve these limits [3]. In this study, silane-modified epoxy resins from pre-hydrolyzed GLYMO was consequently developed in the presence of a relative concentration of 3-aminopropyl triethoxysilane (APTES) and diethylene triamine (DETA) as curing agents. Therefore, hybrid silica sol-gel coatings were prepared with different silica content. The coatings were applied on AA2024 then the effect of the silica content on some properties were investigated.

\section{Materials and methods}

\subsection{Materials}

As the organic phase precursor, DEGBA epoxy resin of EEW=450-500 g/equiv. was obtained from HUNTSMAN (China). The inorganic precursors, GLYMO and APTES were obtained from Sigma-Aldrich, USA. DETA as a curing agent was obtained from $\mathrm{CDH}$, India. Acetone (solvent) as well as $\mathrm{HCl}$ (37 wt\% in water) was purchased from BDH, England. All reagents were used as received without any further modification or processing. All coatings were performed at ambient temperature on freshly prepared coupons of Aluminium alloy 2024-T3 of dimensions $(2.54 \times 10.16 \times 0.156) \mathrm{cm}^{3}$ obtained from Q-panel, USA. Coupons were

\footnotetext{
${ }^{a}$ Corresponding author : m.yossry@mtc.edu.eg
} 
cleaned with deionized water followed by ultrasonic cleaning in acetone and then dried for 30 min at $70{ }^{\circ} \mathrm{C}$ then stored in desiccator till their use.

\subsection{Preparation of silica/epoxy sol-gel coating}

The sol-gel technique depends on a series of hydrolysis and condensation reactions as shown in equations (1-3), as follows:

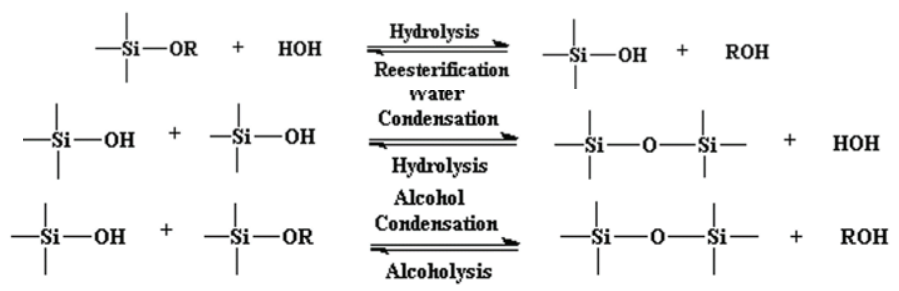

A mixture consisting of epoxy resin and GLYMO in the presence of acetone was prepared according to the wt. ratio of 1:1:6 respectively. The mixture was stirred for $15 \mathrm{~min}$. till the complete dissolution of the epoxy resin. Conc. $\mathrm{HCl}$ was added to the solution preserving a water ratio of 3 with respect to the amount of GLYMO, keeping the $\mathrm{pH}$ within the range of $4.5 \pm 0.3$. The solution was stirred for $1 \mathrm{hr}$. while heating to $100 \mathrm{oC}$ then the solution was left overnight. The solution then divided into six portions in which different amounts of hardeners (DETA and APTES) were to be added according to Table 1. Each portion is to be stirred for $30 \mathrm{~min}$. then spayed over the Al coupons. The thickness of the coat is about $50 \pm 5 \mu \mathrm{m}$.

\subsection{Characterization and measurements}

FTIR analysis was performed using a JASCO 4100 FTIR spectrometer. The surface morphologies of the used coupons were investigated using SEM (Zeiss EVO-10). The contact angle was measured using a ramé-hart goniometer model 250 adopting the sessile drop model with a syringe-out technique. (TGA) studies on the samples were executed on SHIMADZU TGA-50H Analyser. The thickness of the prepared hybrid coat sprayed over the aluminium substrate was to be measured by the coat thickness gauge Kett LZ-330C. The adhesion measurements were performed on double-lap joints with the coat prepared to be used as the adhesive material. A pull-off adhesion test was performed according to ASTM 5656 at room temperature on a GALDABINI QUASAR 100. The Vickers hardness measurements were performed on INSTRON TUKON $2100 \mathrm{~B}$.

\section{Results and discussion}

Table 1. Different compositions of the prepared hybrids with the corresponding mechanical and physical results.

\begin{tabular}{|c|c|c|c|c|c|c|c|}
\hline \multirow[b]{2}{*}{ Sample } & \multirow[b]{2}{*}{$\begin{array}{l}\text { GLYMO:Epoxy } \\
\text { ratio* }\end{array}$} & \multirow[b]{2}{*}{$\begin{array}{c}\text { Hardeners } \\
\text { (APTES:DETA) ratio }\end{array}$} & \multirow{2}{*}{$\begin{array}{l}\text { Shear } \\
\text { stress } \\
\text { (MPa) }\end{array}$} & \multicolumn{2}{|c|}{ Hardness } & \multirow[b]{2}{*}{$\begin{array}{l}\text { Contact } \\
\text { angle }\end{array}$} & \multirow[b]{2}{*}{$\begin{array}{l}\text { Silica Content } \\
\text { (\%) }\end{array}$} \\
\hline & & & & HV & $\begin{array}{c}\text { Mean diagonal } \\
\text { length }(\mu \mathrm{m})\end{array}$ & & \\
\hline A0 & $1: 1$ & $0: 1$ & 1.13 & 147.3 & 354.8 & $69.7^{\circ}$ & $12.12 \%$ \\
\hline $\mathrm{A} 1$ & $1: 1$ & $1: 4$ & 1.67 & 188.8 & 313.4 & $73.6^{\circ}$ & $15.04 \%$ \\
\hline A2 & $1: 1$ & $1: 1.5$ & 1.87 & 221.0 & 289.7 & $74.9^{\circ}$ & $18.31 \%$ \\
\hline A3 & $1: 1$ & $1: 0.67$ & 1.29 & 170.8 & 329.5 & $70.5^{\circ}$ & $21.36 \%$ \\
\hline A4 & $1: 1$ & $1: 0.25$ & 1.20 & 149.1 & 352.7 & $62.5^{\circ}$ & $24.12 \%$ \\
\hline A5 & $1: 1$ & $1: 0$ & 0.92 & 134.4 & 371.5 & $59.6^{\circ}$ & $27.59 \%$ \\
\hline
\end{tabular}

\subsection{FTIR Analysis}

Using the Fourier Transform Infrared technique to investigate the preparation of the hybrid network, the spectra of the main precursors of the hybrid coat were studied individually as shown in Figure 1.

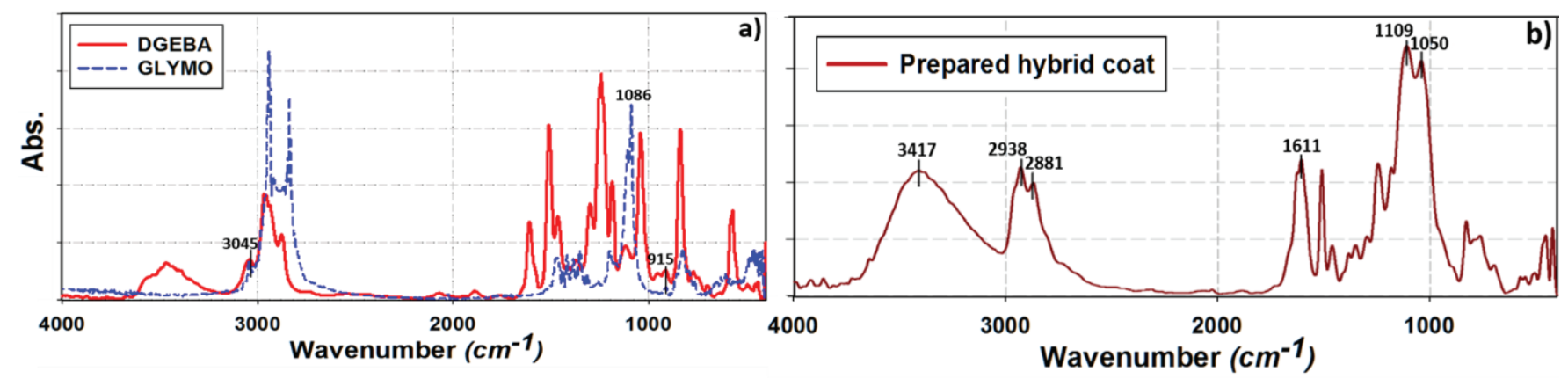

Figure 1. FTIR spectra of a) DGEBA and GLYMO, b) The prepared hybrid coat. 
Primarily, the spectrum of each precursor was investigated and the main structural bands were focused upon as shown in Figure 1.a. Starting with DGEBA (Epoxy), the peaks aiming at both the benzene and the oxirane rings (found around 3045 and $915 \mathrm{~cm}^{-1}$ ) were specified. For the main ceramic-constituent precursor, GLYMO spectrum was clarified for the Si-O-C bands (at $1200-1000 \mathrm{~cm}^{-1}$ ) and the oxirane ring peaks [4-6]. The spectra of the prepared hybrid coats were compared to each other and it was found to be approximately identical. Therefore, a selected spectrum of sample A2 was shown in Figure 1.b as a representative for all hybrid coats. The spectrum was revised for the oxirane ring peaks at $\left(3047\right.$ and $\left.916 \mathrm{~cm}^{-1}\right)$ that have almost diminished. Also, the bands related to the amino groups along with the hydroxyl groups at the ranges of $\left(3570-3200 \mathrm{~cm}^{-1}\right)$ and $\left(1620-1550 \mathrm{~cm}^{-1}\right)$ were highly recorded, indicating the breakage of the oxirane ring and the existence of uncondensed hydroxyl groups with a residual amount of the curing agent. Aside from the oxirane ring, the Si-O-C bands were studied. Once the silica network are created, the sharp peaks are replaced with those broad peaks as found in Figure 1.b at (1050 and $\left.1109 \mathrm{~cm}^{-1}\right)$. Conservatively, a peak at the range of $3420 \mathrm{~cm}^{-1}$ is taking place form the hydrolysed Si-OH species. Also, the peak at $3420 \mathrm{~cm}^{-1}$ may indicates the residuals of the corresponding released alcohol [7,8]. Therefore, the silica network formation through the sol-gel technique is successfully achieved.

\subsection{Mechanical characterization}

\subsubsection{Adhesion test}

Applying different ratios of APTES to DETA to the (Epoxy-GLYMO) base coat, in which APTES is used as another source of silica, has made the synthesis of a silica based hybrid coat more integrated. Appling the test for the prepared combinations, the load-deformation curves were analyzed and the max. load applied as an indication for adhesion strength were assigned. As a result, the shear stress for a double area of $\left(2.5 \times 7 \mathrm{~cm}^{2}\right)$ was concluded as shown in Table 1. As shown, introducing APTES for samples A1 and A2 offered a higher adhesion than that of the DETA only (A0). Then, combinations of higher contribution of APTES to DETA (A3 and A4) have shown a gradual decrease reaching the minimum at APTES only (A5). At first, the addition of APTES produces a more homogeneous, compatible hybrid coat that gradually increases adhesion. A more compatible coat would offer a contribution of the hydrolyzed silanol groups to the bonding with the substrate forming a Si-O-Al bond increasing adhesion. Further increase of APTES, would increase the formed Si-O-Al bonds on the expense of stronger epoxy-metal bonds resulting in a less adherent coat [2]. Also, increasing the ratio of APTES would force the formation of a thin layer of silica (Figure 2) with an unaviodable micro-cracks that further decrease the adhesive power of the hybrid coat [2].

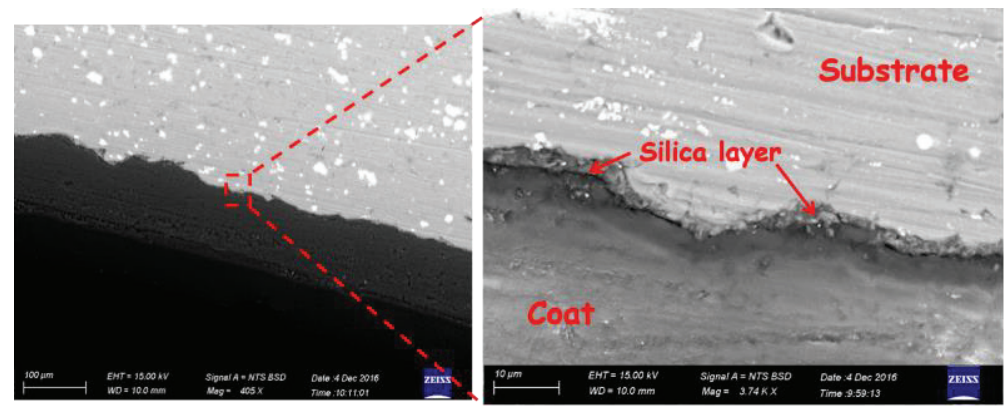

Figure 2. SEM images for a cross-section of the A5 sample showing silica agglomeration over the interface.

\subsubsection{Hardness test}

Applying Vickers hardness test on the HV10 mode to the different hybrids had come with the results listed in Table 1. The results showed an increase in the hardness with the increase in the silica content (adding APTES) reaching a max. at A2. Further addition would form silica particles in clusters. Silica agglomerations would show micro-cracks within the coat affecting largely the hardness [2]. Figure 3 shows SEM images for A4 and A5 revealing the micro-cracks found in silica agglomerations.
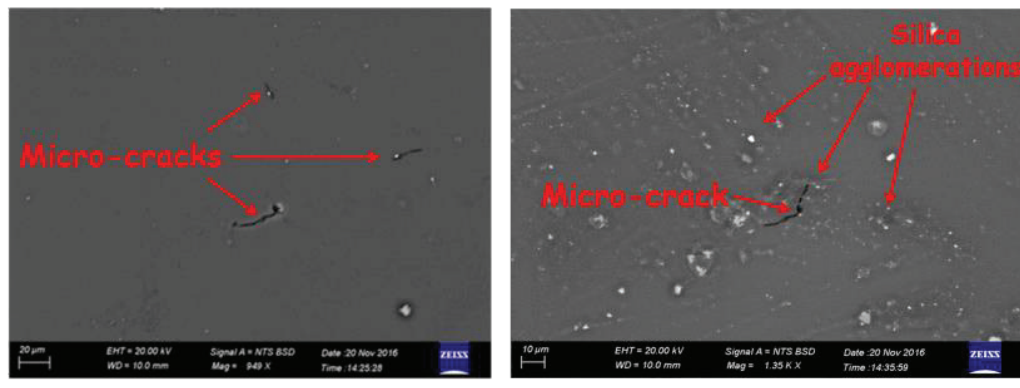

Figure 3. SEM images for A4 and A5 samples' surface showing fine micro-cracks.

\subsection{Contact angle test}


Applying a $1 \mu \mathrm{m}$ distilled water drop, contact angle investigation has come out with the following results showed in Table 1. The results showed that the coat formed is generally hydrophilic and that the wettability of the surface coat varies along the different compositions. It was mentioned that the addition of silica particles would affect the cross-linking density of the hybrid network causing an increase in the free volume as shown in Figure 4. Using silane coupling agents, like GLYMO, would create covalent bonds between networks that decrease the free volume as much as possible [9]. Introducing APTES, would increase the bonding between both networks resulting in a more compact hybrid [10]. The results for (A0, A1, and A2) showed that the more the APTES is added, the more compact the network is, which results in an increase in the contact angle. In other words, the wettability decreases by the addition of the APTES reaching a minimum at the sample A2 then increases again.
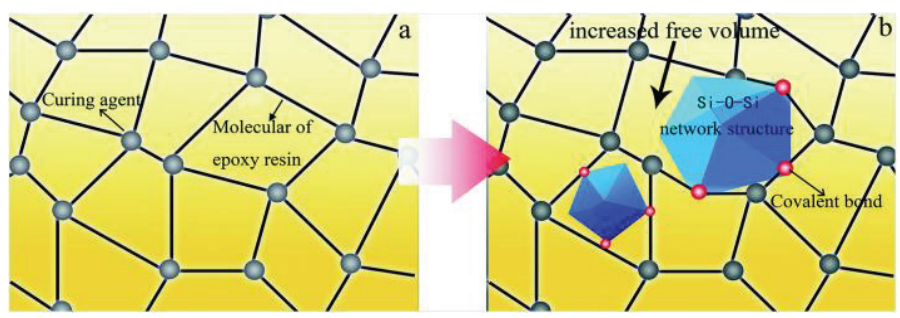

Figure 4. The increase in the free volume due to the presence of silica particles.

Further addition of APTES over the A2 sample, showed a gradual increase in the wettability (decrease in the contact angle) for higher silica content samples (A3, A4, and A5). This could be attributed to the increase of the stresses during the formation and curing of the hybrid network as a result of a high silica percentage [11]. The existence of such stresses would create micro-cracks within the coat that ease the diffusion of water through the coat making the coat more and more hydrophilic.

\subsection{Thermal Gravimetric analysis (TGA)}

TGA is used to evaluate the thermal stability and to determine the silica content involved for each hybrid composition. Combustion of a small amount of each prepared coat in a dynamic airflow from ambient temperature to $800^{\circ} \mathrm{C}$ would give the results shown in Table 1.The silica content for all samples is represented by the remaining weight with respect to that originally introduced (excluding the ash content from the neat epoxy). Figure 5 shows the TGA thermographs of the neat epoxy along with its silica-bonded hybrids. It is clear from the graphs that the neat epoxy sample has shown nearly a complete decomposition while introducing GLYMO had increased the silica content up to $12 \%$. Using different compositions of APTES increases the inorganic content (represented in silica) of the coat. A completely APTES cured sample had shown the max. silica content of $27.6 \%$.
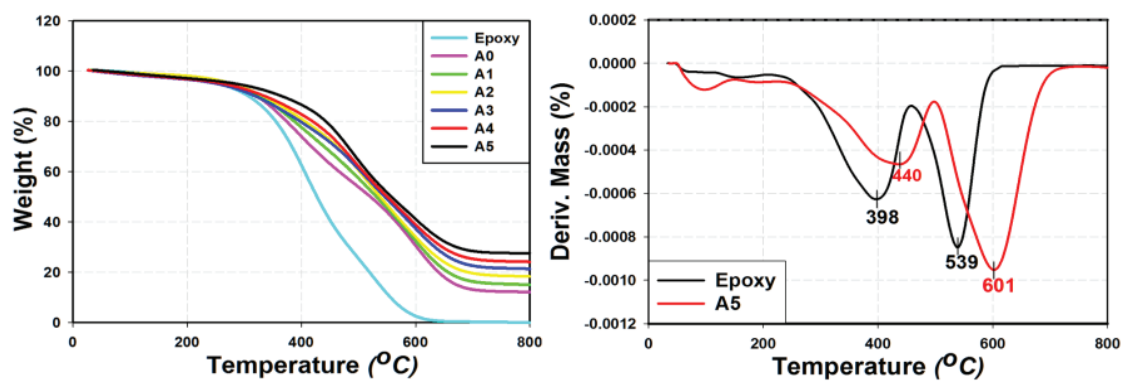

Figure 5. TG/DTG thermographs of the neat epoxy and the hybrid coats.

Reviewing the DTGA thermographs (Figure 5) shows that the thermo-oxidative degradation of epoxy and its hybrids is done over a three-stage process [12]. The curves starts with a small inclination at the range of (120-180oC) that corresponds to the first stage. In this stage, the loss of water adsorbed from the surrounding and that condensed from the residual hydroxyl groups of the silanol groups $(\mathrm{Si}-\mathrm{OH})$ are involved $[2,12,13]$. A second stage is associated to the breakage and the decomposition of the crosslinked organic polymer network in epoxy $[2,12]$. Then, a third oxidative-degradation stage stems from the structural phaseseparation conversion or an oxidation of the aromatic rings of epoxy [14,15].

A comparison of the DTGA of the DETA cured Epoxy and the A5 sample (the APTES cured (Epoxy/GLYMO) hybrid with the max. silica content) shown in Figure 5. It is obvious that a similar decomposition patterns are obtained that confirms the contribution of the organic components only to the weight loss in the hybrid materials. However, all degradation steps were found to occur at slower rates and higher temperatures for the silica-contained hybrids. The increased onset decomposition temperature (Td) from $398 \mathrm{oC}$ to $440 \mathrm{oC}$ is attributed to the insulating barriers created by the heat-resistant nano-silica particles on the matrix that protects the organic matrix from the thermo-oxidative degradation [12]. While the increase in the other decomposition temperature from $539 \mathrm{oC}$ to $601 \mathrm{oC}$ is attributed to the covalent bonds formed between the amino groups linked to the silicanetworks and the epoxy resin that can postpone the breakdown of the aromatic rings [15]. Therefore, the introduction of silica to the thermoset epoxy coat had offered a stability to the all hybrids rather than the neat epoxy $[1,12,16]$.

\section{Conclusions}

Silica-based hybrid coatings prepared by the reaction of DGEBA epoxy resin and GLYMO in the presence of APTES and DETA were prepared and characterized. Confidently, FTIR spectra confirmed the formation of silica domains within the epoxy matrix. It was observed that the adhesion strength has obviously risen with increasing the APTES content to that of the A2 sample 
(APTES:DETA ratio of 1:1.5 by wt.) due to the increase in bonding with the substrate. Also, the same sample has recorded the highest hardness of 221.0 HV compared to other compositions as it contains the max. percentage of silica with no cracks within the coat. On the other side, increasing the silica content was observed to increase the thermal stability of hybrid coat, in which sample A5 (with silica content up to $27.59 \%$ ) has shown higher thermal stability rather than the other hybrids and the neat epoxy. Sample A2 has shown the best-compromised performance among the prepared hybrids.

\section{References}

1. D. Levy and M. Zayat, eds. The Sol-Gel Handbook: Synthesis, Characterization and Applications, 3-Volume Set, (John Wiley \& Sons, 2015)

2. E. Bakhshandeh, A.Jannesari, Z. Ranjbar, S. Sobhani, M. R. Saeb, Prog. Org. Coat. 77, 1169-1183 (2014)

3. S. R. Davis, A. R. Brough, A. Atkinson, J. Non-Cryst. Solids 315, 197-205 (2003)

4. J. Coates, EAC (2006)

5. V. Tagliazucca, Doctoral dissertation, University of Trento (2010)

6. I. M. Šapić, L. Bistričić, V. Volovšek, V. Dananić, Macromol. Symp. 339, 122-129 (2014)

7. W. Zhang, A. A. Dehghani-Sanij, R. S. Blackburn, Prog. Nat. Sci. 18, 801-805 (2008)

8. R. M. Almeida, C. G. Pantano, J. Appl. Phys. 68, 4225-4232 (1990)

9. C. Peng, J. Li, Z. Wu, W. Peng, D. Zhou, RSC Adv. 6, 38300-38309 (2016)

10. S. L. Case, Doctoral dissertation, Virginia Tech. (2003)

11. X-R. Deng, Q-H. Zhang, X-Y. Lei, W. Yang, H-H. Hui, J. Wang, J. Shen, J. Sol-Gel Sci. Technol. 73, 242-249 (2015)

12. Z. Ahmad, F. Al-Sagheer J. Sol-Gel Sci. Technol. 72, 334-343 (2014)

13. J. Z. Zheng, X. P. Zhou, X. L. Xie, Y. W. Mai, Nanoscale 2, 2269-2274 (2010)

14. Y. Liu, Z. Du, C. Zhang, H. Li, Int. J. Polym. Anal. Charact. 11, 299-315 (2006)

15. Z. Xi, H. Qingliang, G. Hongbo, J. Mat. Chem. 1, 2886 (2013)

16. K. Y. Huang, C. J. Weng, S. Y. Lin, Y. H. Yu, J. M. Yeh, J. Appl. Polym. Sci. 112, 1933-1942 (2009) 